NOTES

\title{
Synthesis and Properties of Helical Polyamides Containing Atropisomeric Binaphthyl Units and Crown Ether Rings in the Main Chain
}

\author{
Yuya AgAtA, Motoyasu KobAYAShi, Hiroshi Kimura, and Makoto TAKeISHI ${ }^{\dagger}$ \\ Department of Polymer Science and Engineering, Yamagata University, Yonezawa, Yamagata 992-8510, Japan
}

(Received June 25, 2002; Accepted October 12, 2002)

KEY WORDS Helical Polymer / Polyamide / Crown Ether / Atropisomeric Binaphthyl /

Chiral synthetic polymers are currently attracting much interest. Thus far, according to the particular interest, many types of helical polymers have been synthesized. ${ }^{1}$ Most of helical polymers so far reported, e.g., polymethacrylates, ${ }^{2}$ polyisocyanates ${ }^{3}$ and polyacetylenes, ${ }^{4}$ were prepared by chain reaction polymerization. We tried to synthesize one-handed helical aromatic polyamides by step reaction polymerization, in which bifunctional axially dissymmetric, or atropisomeric, biphenyl or binaphthyl joints were combined with rigid aromatic segments. ${ }^{5,6}$ Optically active polymers having $\mathrm{C}_{2}$-chiral units in the main chain were also prepared by $\mathrm{Pu}$ et $a .^{7}$ and Takata et al. ${ }^{8}$ One-handed helical polymers has recently received considerable attention because of their functions. Okamoto et al. utilized helical polymethacrylates for optical resolution. ${ }^{1}$ $\mathrm{Pu}^{9}$ and Takata et al. ${ }^{10}$ investigated application of such helical polymers to use as asymmetric catalysts. We examined photo-induced transformation of the helical polyamides that contain azobenzene segments to prepare photosensitive materials, ${ }^{5}$ and have studied stereospecific interaction of helical polycations with chiral anions. ${ }^{6}$ Jaycox et al. also reported photosensitive polyamides containing atropisomeric binaphthyl linkages in their backbones and mentioned application of them for optical data storage materials. ${ }^{11}$

By the way, selective incorporation of metal cations into crown ether rings was investigated, ${ }^{12}$ and polyamides containing crown ether moieties in the backbone were reported. ${ }^{13}$ Furthermore, an interesting chiral crown ether that is made up with a chiral binaphthyl derivative has been synthesized to use for optical resolution of chiral ammonium salts. ${ }^{14}$ We are interested in introduction of crown ether rings into helical polymers because a change in the chiral conformation by incorporation of metal ions is anticipated.

In this paper, we describe synthesis of chiral

${ }^{\dagger}$ To whom correspondence should be addressed. polyamides that have atropisomeric binaphthyl joints and crown ether rings in the main chain, and discuss the incorporation of metal ions into the rings.

\section{EXPERIMENTAL}

\section{Materials}

Dimethylformamide (DMF) was purified by distillation over calcium hydride. 1,8-Diazabicyclo[5,4,0]undec-7-en (DBU) was purified by distillation under reduced pressure. Triphenylphosphine was recrystallized from $n$-hexane. Reagent-grade tetrakis(triphenylphosphine) palladium $(0),(R)$ - and $(S)$-2,2'-dihydroxy1,1'-binaphthyl, 1, 1, 1, 3, 3, 3-hexafluoro-2-propanol (HFIP), and othe reagents were used as received.

$(R)$ - and $(S)-6,6^{\prime}$-dibromo-2,2'-diethoxy-1,1'-binaphthyl $((R)$-BEBN, $(S)$-BEBN) were prepared from $(R)$ - and $(S)$-2,2'-dihydroxy-1,1'-binaphthyl according to the method of Deussen et al. ${ }^{15}(R)$-BEBN: mp 160$161^{\circ} \mathrm{C},[\alpha]_{\mathrm{D}}{ }^{25}+17.7^{\circ}\left(c=0.2, \mathrm{CHCl}_{3}\right) ;(S)-1: \mathrm{mp}$ $162-164^{\circ} \mathrm{C}$ (lit. $\left.{ }^{15} 161-162^{\circ} \mathrm{C}\right),[\alpha]_{\mathrm{D}}{ }^{25}-17.4^{\circ}(c=0.2$, $\left.\mathrm{CHCl}_{3}\right)\left(\right.$ lit. $\left.^{15}[\alpha]_{\mathrm{D}}{ }^{25}-17.7^{\circ}\left(c=0.2, \mathrm{CHCl}_{3}\right)\right)$. Transand cis-diaminodibenzo-18-crown-6 (trans-DADBC, cis-DADBC) were prepared by the method of Feigenbaum et al. ${ }^{16}$ trans-DADBC: mp 203-205 ${ }^{\circ} \mathrm{C}$ (lit. ${ }^{16}$ $\left.199-203{ }^{\circ} \mathrm{C}\right) ;{ }^{1} \mathrm{H}$ NMR $\left(270 \mathrm{MHz}, \mathrm{DMSO}-d_{6}\right) \delta 3.70$ $4.00(\mathrm{~m}, 16 \mathrm{H}), 4.64(\mathrm{~s}, 4 \mathrm{H}), 6.05(\mathrm{dd}, J=2.7,9 \mathrm{~Hz}$, $2 \mathrm{H}), 6.23(\mathrm{~d}, 2 \mathrm{H}), 6.62(\mathrm{~d}, 2 \mathrm{H})$, cis-DADBC: $\mathrm{mp} 185-$ $186^{\circ} \mathrm{C}$ (lit. ${ }^{16} 180-184^{\circ} \mathrm{C}$ ).

\section{Polymerization}

In a flask were placed BEBN (0.5 mmol), DADBC $(0.5 \mathrm{mmol})$, tetrakis(triphenylphosphine) palladium $(0)$ $(0.03 \mathrm{mmol})$, triphenylphosphine $(0.12 \mathrm{mmol})$, and DMF $(3 \mathrm{~mL})$. After the atmosphere in the flask was changed by introducing carbon monoxide, the reaction solution was heated to $115^{\circ} \mathrm{C}$. Polymerization was started by adding DBU $(1.1 \mathrm{mmol})$, and the solution 

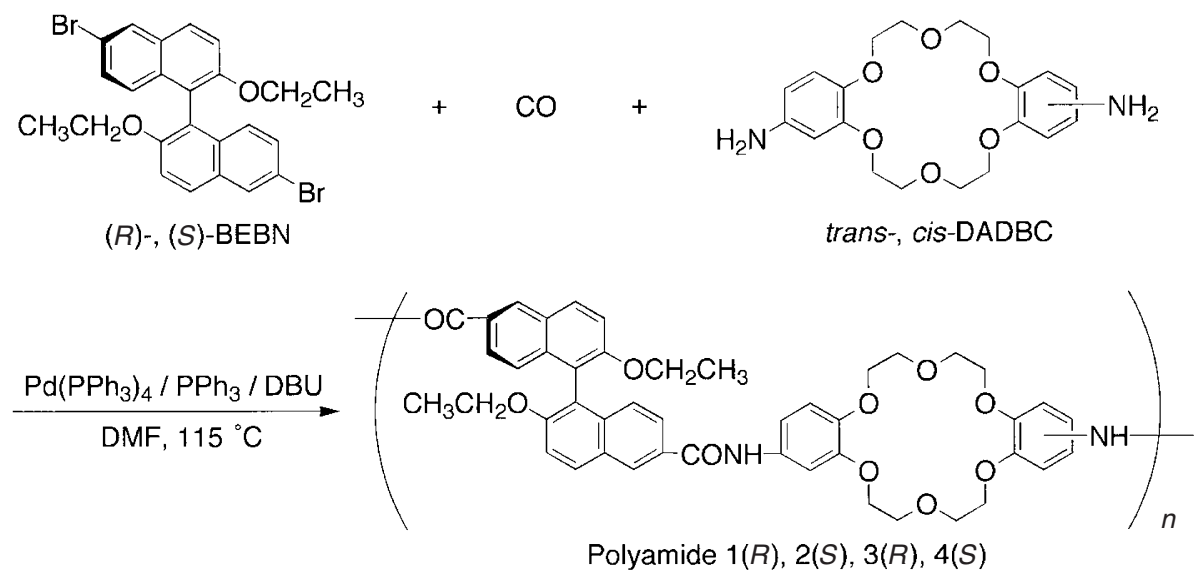

Scheme 1.

Table I. Chiral polyamides containing crown ether ring in the main chain ${ }^{\mathrm{a}}$

\begin{tabular}{cclccrc}
\hline Polyamide & BEBN & DADBC & Yield $(\%)$ & $M_{\mathrm{n}}{ }^{\mathrm{b}}$ & \multicolumn{1}{c}{$M_{\mathrm{w}}{ }^{\mathrm{b}}$} & {$[\alpha]_{\mathrm{D}}{ }^{25}(\mathrm{deg})^{\mathrm{c}}$} \\
\hline $1(R)$ & $(R)-$ & trans - & 72 & 6900 & 12700 & -45.2 \\
$2(S)$ & $(S)-$ & trans - & 72 & 5800 & 9900 & +44.3 \\
$3(R)$ & $(R)-$ & cis- & 80 & 8800 & 19100 & -52.4 \\
$4(S)$ & $(S)-$ & cis- & 90 & 9500 & 20000 & +53.4 \\
\hline
\end{tabular}

${ }^{a}$ The polymerization conditions were presented in the experimental section. ${ }^{\mathrm{b}}$ Determined by GPC with standard polystyrenes in DMF. ${ }^{\mathrm{c} S p e c i f i c}$ rotations were measured in DMSO $\left(\mathrm{c}=0.2 \mathrm{~g} \mathrm{dL}^{-1}\right)$.

was kept at $115^{\circ} \mathrm{C}$ with stirring for $12 \mathrm{~h}$. The resulting polyamide was precipitated in methanol to separate out by filtration. : ${ }^{1} \mathrm{H}$ NMR $\left(270 \mathrm{MHz}, \mathrm{DMSO}-d_{6}\right) \quad \delta$ $1.03(t, 6 \mathrm{H}), 3.85-4.14(\mathrm{~m}, 20 \mathrm{H}), 6.92(\mathrm{~d}, 2 \mathrm{H}), 7.00$ (d, $2 \mathrm{H}), 7.35$ (d, $2 \mathrm{H}), 7.47$ (s, $2 \mathrm{H}), 7.72$ (dd, $J=1.7$, $9 \mathrm{~Hz}, 4 \mathrm{H}), 8.22(\mathrm{~d}, J=9 \mathrm{~Hz}, 2 \mathrm{H}), 8.60(\mathrm{~s}, 2 \mathrm{H}), 10.2$ (s, $2 \mathrm{H})$.

\section{Characterization}

FT-IR spectra were recorded on a HORIBA FT-210 spectrometer. A JASCO DIP-1000 polarimeter was used to measure specific rotations. CD spectra measurements were performed on a JASCO J-720 WI. Molecular weights were determined by GPC using a TOSOH HLC- 8120GPC (Column: TSKgel GMH $\mathrm{HR}^{+}$ TSKgel G2000 H $\mathrm{XL}_{\text {). }}$.

\section{Computational Studies}

To compute the most stable helical conformations of polymers obtained, force field molecular modeling was performed with the MM2 force field implemented by CAChe system.

\section{RESULT AND DISCUSSION}

\section{Helical Conformations}

We synthesized one-handed helical polyamides by reactions of bifunctional atropisomeric biphenyl or binaphthyl derivatives with aromatic diamines, and in- vestigated photo-induced transformation of the helical structure ${ }^{5}$ and stereospecific interaction of cationic one-handed helices with chiral anions. ${ }^{6}$ Similar helical polyamides containing crown ether rings in the main chain were also prepared by the palladium-catalyzed reaction of $(R)$ - or $(S)$-BEBN, carbon monoxide, and trans- or cis-DADBC in the present work (Scheme 1). Number-average molecular weights of the polyamide obtained were 5.8-9.5 $\times 10^{3}$, as shown in Table I. The specific rotation of the polymer was about three times as large as that of the axially dissymmetric starting binaphthyl reagent, probably because of the change of bromine substituents of BEBN into polar amide. 3(R) and $4(S)$ obtained with $c i s$-DADBC have somewhat larger molecular weights and specific rotations than $1(R)$ and 2(S) obtained with trans-DADBC. It is known that specific rotation of chiral polymers increases with increasing molecular weight; ${ }^{17}$ however, with these a few data, the change in specific rotations can not be related to the molecular weights. The specific rotations of the polymers in Table I are rather small compared with optically active helical polymers so far reported. This would be ascribed to the flexible structure of the helix because of the ether ring in the main chain. All the polymers were soluble in polar solvents such as DMF and dimethylsulfoxide (DMSO), but insoluble in less polar tetrahydrofuran and chloroform.

Figure 1 presents the CD spectra of polyamide $1(R), 2(S)$, and $3(R)$. The mirror imaged CD spec- 
Table II. Changes in specific rotation of helical polyamides on addition of alkaline metal salts

\begin{tabular}{ccccccc}
\hline \multirow{2}{*}{ Run } & \multirow{2}{*}{ Polyamide } & \multicolumn{5}{c}{$[\alpha]_{\mathrm{D}}{ }^{25}(\mathrm{deg})^{\mathrm{a}}$} \\
\cline { 3 - 7 } & & blank & $\mathrm{Li}^{+}$ & $\mathrm{Na}^{+}$ & $\mathrm{K}^{+}$ & $\mathrm{Cs}^{+}$ \\
\hline 1 & $1(R)$ & -45.2 & -30.0 & -36.6 & -18.7 & -34.4 \\
2 & $3(R)$ & -52.4 & -34.8 & -25.3 & -39.8 & -53.4 \\
$3^{\mathrm{b}}$ & $3(R)$ & -141.0 & -107.0 & -76.0 & $-^{\mathrm{c}}$ & $-^{\mathrm{c}}$ \\
\hline
\end{tabular}

aPolymers: $1.27 \times 10^{-3} \mathrm{M}$ (based on the ether ring), perchlorate salts: $1.27 \times 10^{-2} \mathrm{M}$; specific rotations were measured in DMSO $\left(c=0.1 \mathrm{~g} \mathrm{dL}^{-1}\right)$. ${ }^{\mathrm{b}}$ In HFIP-water (vol. 5:1). ${ }^{\mathrm{c}}$ Perchlorate salts are insoluble in HFIP-water.

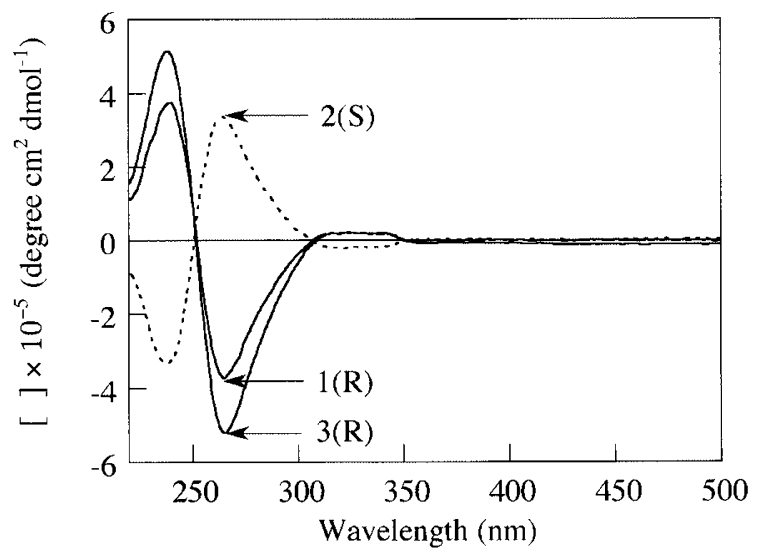

Figure 1. CD spectra of $1(R), 2(S)$, and $3(R)$ in HFIP.

tra of $1(R)$ and $2(S)$ indicate that these polymers have symmetric structures to each other. The more enhanced Cotton effect was observed for $3(R)$ containing cis-DADBC moieties than for $1(R)$ containing transDADBC, being related to their specific rotations. Since the polyamides obtained have axially dissymmetric binaphthylene joints in the main chain, they have onehanded helical structures; therefore, the most stable conformations of the polyamides were computed with CAChe system. The profile of the helical conformation of $3(R)$ is presented as a top view and a side view in Figure 2. However, a helix-like conformation is allowed only at very low temperatures for such a flexible polymer because of its molecular motion in a solution.

\section{Complexation with Metal Ions}

Pedersen reported the synthesis of crown ethers for the first time and their remarkable complex formation with metal salts in organic solvents attracted particular interest. ${ }^{12}$ Ever since analogous multidentate cyclic compounds have been widely studied and very diverse application of them have emerged. ${ }^{18}$ Polymeric crown ethers have also been received considerable attention: Feigenbaum et al. synthesized a polyamide that contains crown ether rings in its main chain, ${ }^{16}$ Kopolow et al. reported polystyrene derivatives that have such ring pendants, ${ }^{19}$ and a synthetic method of a chiral conjugated crown ether polymer was first shown by $\mathrm{Pu}$ et $a .^{20}$ We also synthesized chiral helical polyamides having crown ether rings in the main chain, ${ }^{21}$ and in the

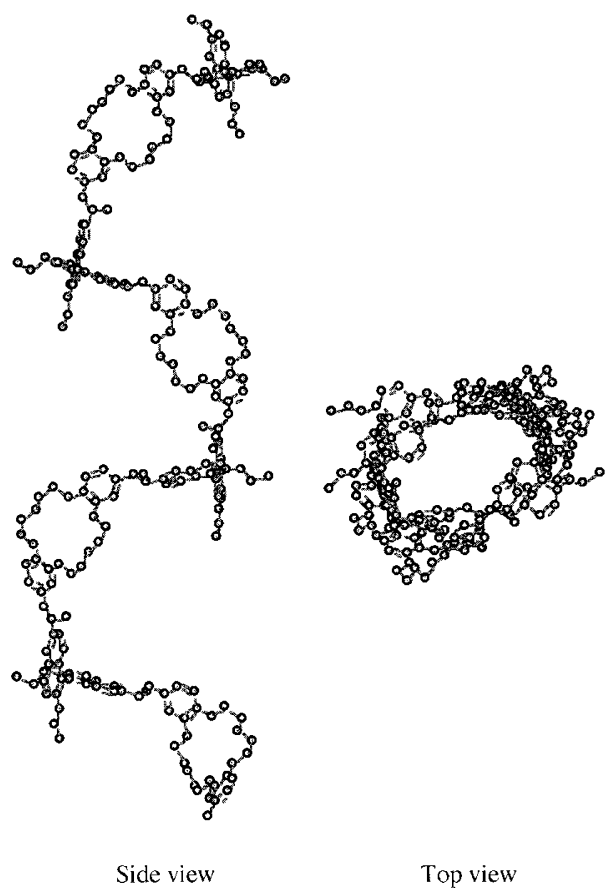

Figure 2. The most stable conformation of polyamide 3(R) optimized with the MM2 force field implemented by CAChe system.

present study the complex formation of the ether rings with alkali metal ions was examined. Table II shows changes in specific rotations of the polyamides on addition of alkali metal salts. In the case of $1(R)$ with transDADBC moieties, addition of potassium salt resulted in the most definite change in the specific rotation. Specific rotations of optically active compounds depend largely on polarity of the media; consequently, the incorporation of metal is presumed to cause a change in the specific rotation. Above observations are consistent with well known facts that, in usual cases, a potassium ion fits most easily into the ether ring of 18-crown-6, and the complexation constant of the monovalent cation for the ether ring, therefore, is the largest compared with those of other alkaline metal ions. Nevertheless, in the case of 3(R) with cis-DADBC moieties, addition of sodium salts caused the largest change in the specific rotation. Substituent effects of dibenzo-18-crown6 on the selectivity of metal ions have been reported. ${ }^{22}$ This is probably due to deformation of the ether ring; hence, it seems that similar deformation of the ether 


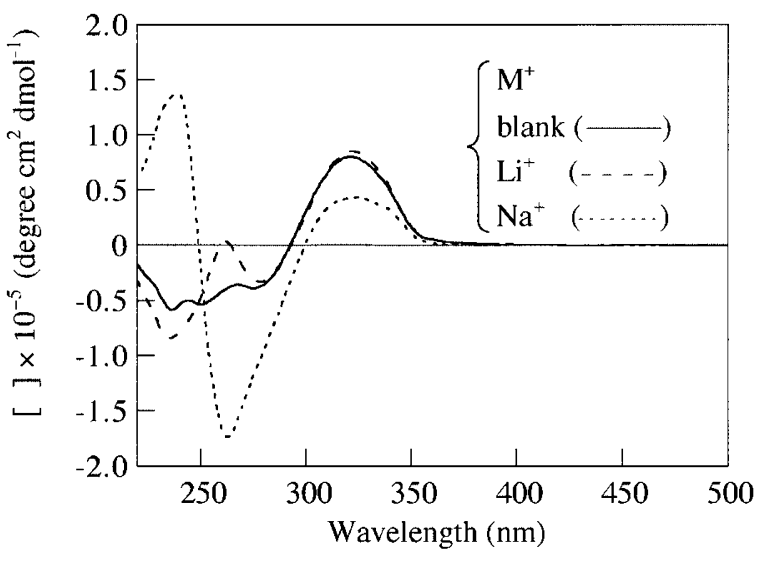

Figure 3. Changes in CD spectrum of $3(R)$ in the presence of alkaline metal ions in HFIP-water (vol. $5: 1) .3(R): 6.35 \times 10^{-5} \mathrm{M}$ (based on the ether ring), perchlorate salts: $6.35 \times 10^{-4} \mathrm{M}$

ring in $3(R)$ leads to reversed complexation constants of sodium and potassium ions. In the cases of lithium and cesium ions that are difficult to complex with the ether ring, the changes in specific rotation are mainly ascribed to changes in polarities of the solvent due to the presence of the salts. The specific rotation of $3(R)$ was observed also in HFIP-water (vol. 5:1) because CD spectra were obtained in this solvent system as described bellow. The negative values of the specific rotation were grater than those in DMSO because of the change in the polarity of the media as mentioned above (Run 3 in Table II).

To estimate the degree of transformation of the helical conformation by the complexation, the change in CD spectrum of $3(R)$ on addition of alkaline metal salts was observed. The changes were too small to observe in DMSO, in which the polymer and all the perchlorate salts are soluble. This indicates that the much polar solvent can solvate both cations and anions of the salts to suppress the complexation that causes deformation of the polymer conformation, which leads to the change in the CD spectrum. Some solvent systems were examined and a pronounced change in the $\mathrm{CD}$ spectrum of $3(R)$ was observed in HFIP- $\mathrm{H}_{2} \mathrm{O}$ (vol. $5: 1$ ) when sodium perchlorate was added (Figure 3 ). This is ascribed to the change in the dihedral angle within the atropisomeric binaphthyl group that was the result of the conformational change of the polymer caused by the complexation. Since potassium and cesium perchlorates are insoluble in the solvent system, the effect of the complexation of the metal ions on the change in CD spectrum could not be observed.

Solution viscosities of polyelectrolytes are relatively large because they adopt expanded random coil conformations due to electrostatic repulsion of the charges on the polymer chain. The complexation of the ether ring-containing polyamides with metal ions is expected

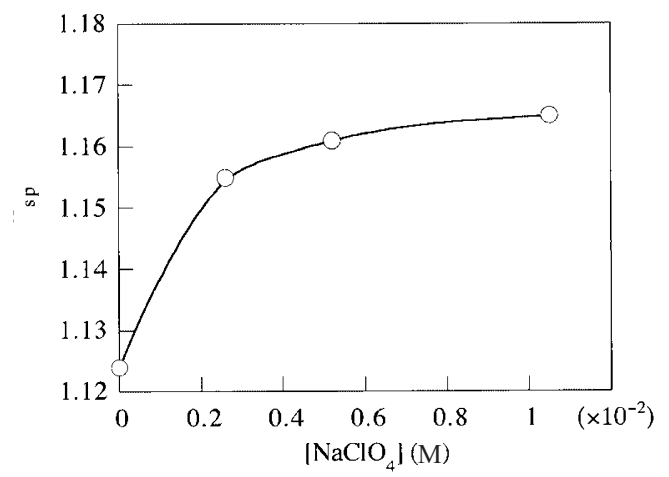

Figure 4. Change in solution viscosity of $4(S)$ in the presence of $\mathrm{NaClO}_{4}$ in HFIP-water (vol. $5: 1$ ) at $25^{\circ} \mathrm{C}$. Polymer concentration: $0.1 \mathrm{~g} \mathrm{dL}^{-1}\left(1.3 \times 10^{-3} \mathrm{M}\right.$ based on the repeating unit).

to confer such a property of polyelectrolytes on the polymer. As shown in Figure 4, the specific viscosity of $3(R)$ in the HFIP-water (vol. 5:1) increased as added sodium perchlorate increased. This arises from extended conformation of the polyamide that carries positive charges of the incorporated metal ions. This conformational change allows the flexible polyamide to assume a helical structure and brings about the CD spectral change because the dihedral angle within the atropisomeric binaphthyl group changes. These observations of solution viscosity also support the complexation.

As described above, Cram's group synthesized chiral crown ethers using atropisomeric binaphthol and investigated chiral recognition by the crown ethers to use them for optical resolution. ${ }^{14}$ We have already prepared one-handed helical polycations and investigated stereospecific, or enantioselective interaction of them with chiral anions, ${ }^{6}$ and are now interested in application of the one-handed helical crown ether polymers to chiral recognition. Hence, further investigations are in progress.

\section{CONCLUSIONS}

Chiral polyamides containing axially dissymmetric binaphthyl twists and crown ether rings in their main chain were synthesized by the palladium-catalyzed reaction of atropisomeric $(R)$ - or $(S)$-BEBN, carbon monoxide, and trans- or cis-DADBC. Incorporation of monovalent metal ions into the crown ether rings in the main chain caused conformational changes of the helical polyamides, which was revealed by changes in $C D$ spectra of them. This interaction is expected to be used for chiral recognition.

Acknowledgments. This work was supported by Grant-in-Aid Scientific Research from the Ministry of Education, Culture, Sports, Science and Technology, 
Japan (No. 13650926).

\section{REFERENCES}

1. T. Nakano and Y. Okamoto, Chem. Rev., 101, 4013 (2001).

2. T. Nakano and Y. Okamoto, Chem. Rev., 94, 349 (1994).

3. M. M. Green, R. A. Gross, C. Crosby III, and R. C. Schilling, Macromolecules, 20, 992 (1987).

4. E. Yashima, T. Matsushima, and Y. Okamoto, J. Am. Chem. Soc., 117, 11596 (1995).

5. a) F. Kondo, M. Hidaka, S. Kakimi, H. Kimura, and M. Takeishi, Polym. Prepr., (Am. Chem. Soc., Div. Polym. Chem.), 38(2), 209 (1997).

b) F. Kondo, D. Takahashi, S. Kakimi, H. Kimura, and M. Takeishi, Polym. J., 30, 161 (1998).

c) F. Kondo, S. Kakimi, H. Kimura, and M. Takeishi, Polym. Int., 46, 339 (1998).

6. Y. Agata, M. Kobayashi, H. Kimura, and M. Takeishi, Polymer, 43, 4829 (2002).

7. a) Q.-S. Hu, D. Vitharana, G. Liu, V. Jain, M. W. Wagaman, L. Zhang, T. R. Lee, and L. Pu, Macromolecules, 29, 1082 (1996).

b) L. Ma, Q.-S. Hu, D. Vitharana, C. Wu, C. M. S. Kwan, and L. Pu, Macromolecules, 30, 204 (1997).

c) L. Pu, Macromol. Rapid. Commun., 21, 795 (2000).

8. T. Takata, Y. Furusho, K. Murakawa, T. Endo, H. Matsuoka, T. Hirasa, J. Matsuo, and M. Sisido, J. Am. Chem. Soc., 120, 4530 (1998).

9. L. Pu, Chem. Eur. J., 5, 2227 (1999).
10. Y. Furusho, T. Maeda, T. Takeuchi, N. Makino, and T. Takata, Chem. Lett., 1020 (2001).

11. S. R. Lustig, G. J. Everlof, and G. D. Jaycox, Macromolecules, 34, 2364 (2001).

12. C. J. Pedersen, J. Am. Chem. Soc., 89, 7017 (1967).

13. W. M. Feigenbaum and R. H. Michel, J. Polym. Sci., A-1, 9, 817 (1971).

14. E. P. Kyba, M. G. Siegel, L. R. Sousa, G. D. Y. Sogah, and D. J. Cram, J. Am. Chem. Soc., 95, 2691 (1973).

15. H. J. Deussen, E. Hendrickx, C. Boutton, D. Krog, K. Clay, K. Bechgaar, A. Persoon, and T. Bjørnholm, J. Am. Chem. Soc., 118, 6841 (1996).

16. W. M. Feigenbaum and R. H. Michel, J. Polym. Sci., A-1, 9, 817 (1971).

17. G. Wulff, U. Zweering, and S. Gladow, Polym. Prepr., (Am. Chem. Soc., Div. Polym. Chem.), 37(2), 448 (1996).

18. a) R. M. Izatt and J. J. Christensen, Ed., "Synthetic Multidantate Macrocyclic Compounds", Academic Press, Inc., New York, N.Y., 1978.

b) F. Vögtle, Ed., "Host Guest Complex Chemistry I", Springer-Verlag, GmbH \& Co., Berlin, 1981.

19. S. Kopolow, T. E. Hogen Esch, and J. Smid, Macromolecules, 4, 359 (1971).

20. H. Cheng, L. Ma, Q.-S. Hu, X.-F. Zheng, J. Anderson, and L. $\mathrm{Pu}$, Tetrahedron: Asymmetry, 7, 3083 (1996).

21. Y. Agata, H. Kimura, and M. Takeishi, Polym. Prepr. Jpn., 48, 256 (1999).

22. K. H. Pannel, W. Yee, G. S. Lewandos, and D. C. Hambrick, J. Am. Chem. Soc., 99, 1457 (1977). 